certain genes (homozygous deficiencies) on the early development of Drosophila.

Several reports dealt with gene effects on pro. cesses which are chemically more or less defined. Thus we had further chapters in the important and rapidly developing researches of Price, Lawrence and others on flower pigments, and Beadle, Ephrussi and others on Drosophila eye colours. Perhaps the most important new contribution in this field was a fresh study by Sewall Wright of guinea pig coat colours, which he dealt with in a preliminary way many years ago. He was now able to suggest a scheme for the relations of the numerous genes which are known, and to present a mathematical theory of their quanti. tative interactions.

Quite a different method of approach to the same problem was reported by Schultz and Caspersson, who, by studying the chromosome itself, obtained data which allow of some speculation as to the chemical changes occurring under the immediate influence of genes. Owing to their strong absorption in the middle ultra-violet, the distribution and changes of the substances belonging to the nucleotide group can be followed in the living cell by spectrophotometric methods elaborated by Caspersson. It is found that in the nucleus the nucleic acids seem to be indispensable for the development of chromosomes, and that they are synthesized mainly in earliest prophase just before the chromosomes split. Schultz studied translocations of parts of the heterochromatic regions of the chromosomes, which are rich in nucleic acid, into normal euchromatic regions in Drosophila. In certain of these translocations the adult flies show variegation for characters affected by genes near the position of the break in the chromosome, as though frequent mutations of the genes had occurred in late stages of development. Schultz showed that this variegation is correlated with cytological effects in the salivary chromo. some bands corresponding to the variegated genes; these effects ranged from appearance of excess nucleic acid to the assumption of a heterochromatic character and finally apparent dis. appearance. It is suggested that the appearance of excess nucleic acid is correlated with an inactivation of the gene as a developmental agent. The nucleic acid metabolism can be followed somewhat further, since it is found that the presence of extra heterochromatic material (for example, supernumerary $Y$-chromosomes) affects both the degree of variegation, the cytological correlates of variegation mentioned above, and also the nucleic acid content of the cytoplasm. The different types of nucleic acid occurring in chromosomes, nucleolus and cytoplasm can be determined spectroscopically and suggestions made as to their functional relations with the fundamental processes of gene action and gene reduplication.

It is possible, as Schultz suggests, that we may in this way obtain some insight into the changes of gene activity in different chromosome regions in the different tissues, which may provide a mechanism for the primary differentiation of the nuclei in development.

\title{
ANIMAL BREEDING IN THE LIGHT OF GENETICS
}

\author{
By DR. J. E. Nichols
}

$\mathrm{T}$ HE opening session was devoted to general considerations of animal genetics. H. C. McPhee described the United States Department of Agriculture's long-term inbreeding projects with pigs, sheep, and poultry, and the emphasis now being laid upon measuring the more complex physiological characters; and in reviewing the work at Wiad, Sweden, G. Bonnier mentioned the economy in numbers of experimental animals made possible by the use of monozygotic twins, and the similarity to plant breeding techniques now being obtained with poultry, where the mixed sperm from many males can be used in artificial insemination. W. K. Hirschfeld and G. M. van der Plank struck notes for many succceding speakers in directing attention to the distinction between frugal and less frugal breeds and families relative to production, and to the need of precise studies on differential demands upon the components of a ration. Their advocacy of the progeny test was pressed further by A. I. Hagedoorn, who suggests restricting to élite 
'nuclei' of breeding stock the function to be sole progenitors of their kind.

The section got into its stride of discussion on August 24 in an all-day session on live-stock improrement in tropical and relatively low nutritional plane conditions. J. H. R. Bisschop gave a comprehensive account of the interesting zootechnical studies on Afrikaner, imported, and grade cattle being conducted in South Africa, with the objectives of defining a type and performance suited to semi-arid conditions and of assessing the degeneration of the pure imported types therein. Using the Australian Merino sheep as an example, J. E. Nichols discussed the practical difficulties of adjusting type to environment when artificial selection is heaviest in more favoured and natural selection in poorer conditions, where commercial production is expected. A. O. Rhoad described the experimental determination of adaptability of cattle to unfavourable conditions in America by means of body temperature reactions, and revealed genetic differences in respect of physiological characters; while Manresa and his collaborators demonstrated significant differences in the hiemo. globin and other constituents of the blood in cattle in relation to atmospheric temperatures (and thereby to adaptability to the local environment) in the Philippines. The economic and social backgrounds of breeding problems in the animal industries in countries from the Gold Coast to Madras were presented in several communications, and R. B. Kelley lodged a strong claim for the importance of ecological and economic studies as prerequisite surveys to constructive breeding policies in tropical Australia. Later, G. Pontecorvo stressed the influence of agricultural conditions on policies of selection in beef and draft cattle in Tuscany.

The use of artificial insemination as a rational technique in animal breeding was discussed in a special session at which were described the methods employed, and their accompanying problems, in Italy, the United States, Rumania, Kenya, and at Lwow. The necessity of adequate criteria of sperm efficiency was strongly stressed by J. Edwards and $\mathrm{A}$. Walton in presenting an account of recent investigations at Cambridge in which the respira. tion rate of sperm has taken a prominent and promising position.

Statistical safeguards and interpretations in genetical experiments with animals and in the analysis of records of performance and progeny tests were discussed notably by F. Yates and J. L. Lush. not only in formal papers, but also in relation to other contributions, especially those on the inheritance of milk yield. This problem, of great practical importance, stimulated one of the most effective discussions of the section, in which particular attention was given to the question of persistency in lactation. The lability of the several phases of a lactation in response to environmental influences of nutrition, management, etc., leads to difficulties in evaluation of records, but empha. sizes the need for detailed analysis as well as ultimate resolution in terms of the physiological processes affecting the component parts of the curve.

In other sessions lethal factors and defects in large animals and genetical characters in small animals were discussed; F. W. Dry presented a case of linkage between horns and abundance of halo hairs in New Zealand Romney sheep, and R. C. Bamber an interesting instance of the operation of a time factor in the inheritance of white spotting in cats.

Breed differences and the recognition of the physiological substrata received further prominence in the session on poultry geneties when F. B. Hutt presented evidence of differential reactions and performances, A. W. Greenwood advanced indications of a genetical control of egg-laying persistency, and A. Ghigi's contribution on cerebral hernia in the fowl evoked discussion on the relationships of skull form, 'crest', and hernia.

Again, in the sessions concerned with nutrition and genetics, and with pig breeding, the same fundamental approach was manifested; as in the demonstration by C. P. MoMeekan and J. Hammond that the plane of nutrition exerts a profound effect upon not only general form but also the growth components so that the direction, as well as the possibility, of selection is influenced by environmental thresholds. That this aspeet of the problem of livestock improvement deserves further scientifically determined discrimination at all stages of growth and in regard to practical breeding policies was emphasized by H. P. Donald and by H. R. Davidson.

It may be said, in fact, that the central theme throughout the proceedings of this section was the evaluation of genetic differences and responses to environmental conditions in relation to the requirements of practical husbandry. 